\title{
Phase Diffusion in Single-Walled Carbon Nanotube Josephson Transistors
}

\author{
Yong Zhang ${ }^{\S}$, Gang Liu, and Chun Ning Lau $(\bowtie)$ \\ Department of Physics \& Astronomy, University of California, Riverside, CA 92521, USA \\ ${ }^{\S}$ Present address: School of Physical Science \& Technology, Southwest University, Chongqing 400715, China \\ Received: 12 May 2008/ Revised: 1 July 2008/Accepted: 6 July 2008 \\ CTsinghua Press and Springer-Verlag 2008
}

\begin{abstract}
We investigate electronic transport in Josephson junctions formed by individual single-walled carbon nanotubes coupled to superconducting electrodes. We observe enhanced zero-bias conductance (up to $10 e^{2} / h$ ) and pronounced sub-harmonic gap structures in differential conductance, which arise from the multiple Andreev reflections at superconductor/nanotube interfaces. The voltage-current characteristics of these junctions display abrupt switching from the supercurrent branch to the resistive branch, with a gate-tunable switching current ranging from $65 \mathrm{pA}$ to $2.5 \mathrm{nA}$. The finite resistance observed on the supercurrent branch and the magnitude of the switching current are in good agreement with the classical phase diffusion model for resistively and capacitively shunted junctions.
\end{abstract}

\section{KEYWORDS}

Carbon nanotubes, Josephson junctions, quantum dot, electrical transport, supercurrent

Carbon nanotubes have emerged as a new model system for quantum dots, as they enjoy several advantages compared with traditional ones based on two-dimensional electron gas systems. For instance, a short nanotube device has a relatively large single particle level spacing $\Delta E$ compared to the charging energy $E_{c}$ a parameter regime difficult to attain by traditional methods. Nanotubes can be readily coupled to different electrode materials such as superconductors [1-11] and ferromagnets [12, 13], thus enabling investigation of transport of spin and Cooper pairs through a 1-D nanostructure. Recently it has been demonstrated that individual single-walled and multi-walled carbon nanotubes (SWNT and MWNT) can function as gate-tunable supercurrent transistors, with typical critical currents of a few nA [5, 7-9]. These experimental results have inspired much interest in the application of SWNT-based Josephson junctions (JJs) as a potential building block for quantum computing architectures based on superconductors. Such JJs also constitute an experimental platform for investigating the intricate interplay among several energy scales such as $E_{\mathrm{c}^{\prime}}$ the thermal energy $k_{\mathrm{B}} T$, the Josephson coupling energy $E_{\mathrm{J}}$ and the Kondo energy, where $k_{\mathrm{B}}$ is Boltzmann constant.

In traditional and nanotube JJs, the thermal and electromagnetic environments play an important role in determining the junction dynamics, which is usually described within the resistively and capacitively shunted junction (RCSJ) model. Here

Address correspondence to lau@physics.ucr.edu 
the phase difference across the junction is visualized as a particle localized in, or running down, the tilted washboard potential, corresponding to the supercurrent-carrying state and the resistive state of the junction, respectively. For short ballistic SWNT JJs, the superconductors are coupled via a single quantized energy level; in the strong Josephson coupling regime $E_{\mathrm{J}} \gg k_{\mathrm{B}} T$, these devices behave as underdamped junctions, with premature switching from supercurrent to resistive branches induced by the electromagnetic environments [5]. In contrast, in the intermediate to weak Josephson coupling regime $E_{\mathrm{J}}<\sim k_{\mathrm{B}} T$, long diffusive MWNT JJs can be described by phase diffusion down the tilted washboard potential [9]. Phase diffusion has also been observed in SWNTs with considerable charging energies, where " $0-\pi$ " transitions in critical currents were inferred [11].

In this paper, we report measurements on short ballistic SWNT devices that are well-coupled to the electrodes (hence with negligible charging energies): we observed Fabry-Perot interference, multiple Andreev reflections (MAR), and gate-tunable voltage-current $(V-I)$ characteristics. Each $V-I$ curve exhibits a sharp switching from the supercurrent to the quasiparticle branches, and the measured switching current ranges from $65 \mathrm{pA}$ to $2.5 \mathrm{nA}$. In contrast to a previous report in Ref. [5], we find that both the magnitude of the switching current and the finite resistance observed in the supercurrent branch are in good agreement with the classical phase diffusion model in the weak Josephson coupling regime $[9,14-16]$. Our results demonstrate that (quasi)ballistic, weakly coupled SWNT Josephson transistors are strongly affected by dissipation determined by the small impedance of the biasing circuit at high frequencies. We also note that our results are obtained at $300 \mathrm{mK}$, indicating that ultralow temperatures are not required for observing supercurrents in SWNT JJs.

The SWNTs were prepared by a chemical vapor deposition method on highly doped $\mathrm{Si} / \mathrm{SiO}_{2}$ substrates [17], and located with respect to the predefined alignment marks using an atomic force microscope. The palladium (Pd)/aluminum (Al) electrodes were fabricated by standard electron beam lithography. Only devices with room temperature resistance below $30 \mathrm{k} \Omega$ were selected for the measurements in a ${ }^{3} \mathrm{He}$ refrigerator. This paper presents results from two different devices: Device 1 with a Pd $(6 \mathrm{~nm}) / \mathrm{Al}(70 \mathrm{~nm})$ bilayer, and source-drain separation of $390 \mathrm{~nm}$; Device 2 with a Pd $(3 \mathrm{~nm}) / \mathrm{Al}$ $(70 \mathrm{~nm})$ bilayer, and separation of $580 \mathrm{~nm}$. The gate dependence of room temperature resistance indicates that both SWNTs are small band gap semiconductors.

Before investigating the superconducting behavior of our superconductor / SWNT / superconductor (S/SWNT/S) junctions, the samples were first characterized in the normal state by applying a magnetic field $H$ of $8 \mathrm{~T}$ which suppresses superconductivity in the $\mathrm{Al}$ electrodes ${ }^{1}$. Figure 1(a) shows a plot of the differential conductance (color) of Device 1 as a function of source-drain voltage $V$ (vertical axis) and gate voltage $V_{\mathrm{g}}$ (horizontal axis). The distinct "checker board" pattern, i.e., the sinusoidal oscillation of the differential conductance of the device with both gate and bias voltages, arises from the so-called Fabry -Perot (F-P) interference [18] of incident and multiply reflected electron waves between two partially transmitting electrodes, or equivalently, from resonant and off-resonant transmission across quantized single particle levels. From Fig. 1(a), we can conclude that the SWNT/ electrode contact is highly transparent, since the device conductance ranges from $1.8 e^{2} / h$ to $3.4 e^{2} / h$, approaching the theoretical limit of $G_{0}=4 e^{2} / h=(6.5$ $\mathrm{k} \Omega)^{-1}$ for a perfectly contacted SWNT. Moreover, the characteristic voltage scale, indicated by the arrow in Fig. 1(a), is $V_{\mathrm{c}} \approx 5.6 \mathrm{mV}$. The energy scale $e V_{\mathrm{c}}=$ $h v_{\mathrm{F}} / 2 L$ corresponds to a $2 \pi$ modulation in the phase accumulated by an electron in completing a roundtrip between two scatterers separated by distance $L$. Here $v_{\mathrm{F}} \approx(0.91 \pm 0.1) \times 10^{6} \mathrm{~m} \cdot \mathrm{s}^{-1}$ is the Fermi velocity of charges in the nanotubes $[19,20]$. The value of $L$ obtained from this estimate, $L \approx 350 \mathrm{~nm} \pm 40 \mathrm{~nm}$, is in good agreement with the source-drain spacing of 390 $\mathrm{nm}$, indicating that scatterings primarily occur at the

\footnotetext{
${ }^{1}$ The critical field of $\mathrm{Al}$ is $10 \mathrm{mT}$. A field of $8 \mathrm{~T}$ was applied for experimental convenience; we did not expect or observe any changes in electrical conductance resulting from the large field, $H$.
} 
nanotube-electrode interface, and are not caused by defects. Thus, our SWNT devices can be considered to be relatively free of defects and to have almost ohmic contacts. For Device 2, a similar interference pattern was also observed with an average conductance around $2 e^{2} / h$, albeit not as periodic as Device 1 . Nevertheless, the dominant energy scale for Device 2 was determined to be $3.4 \mathrm{mV}$, corresponding to $L \approx$ $550 \mathrm{~nm} \pm 60 \mathrm{~nm}$, close to the source-drain spacing of $580 \mathrm{~nm}$. This suggests the nanotube in Device 2 is quasiballistic with the presence of only a few scatterers.

We now focus on the device behavior with superconducting electrodes at $H=0$. At $|V| \geqslant 50 \mu \mathrm{V}$, transport was dominated by the quasiparticles and the $\mathrm{F}-\mathrm{P}$ interference pattern persisted. At small biases $|V| \leqslant 50 \mu \mathrm{V}$, the transport characteristics in both devices changed dramatically: conductance peaks were observed, persisting through all gate voltage ranges, indicating enhanced transport

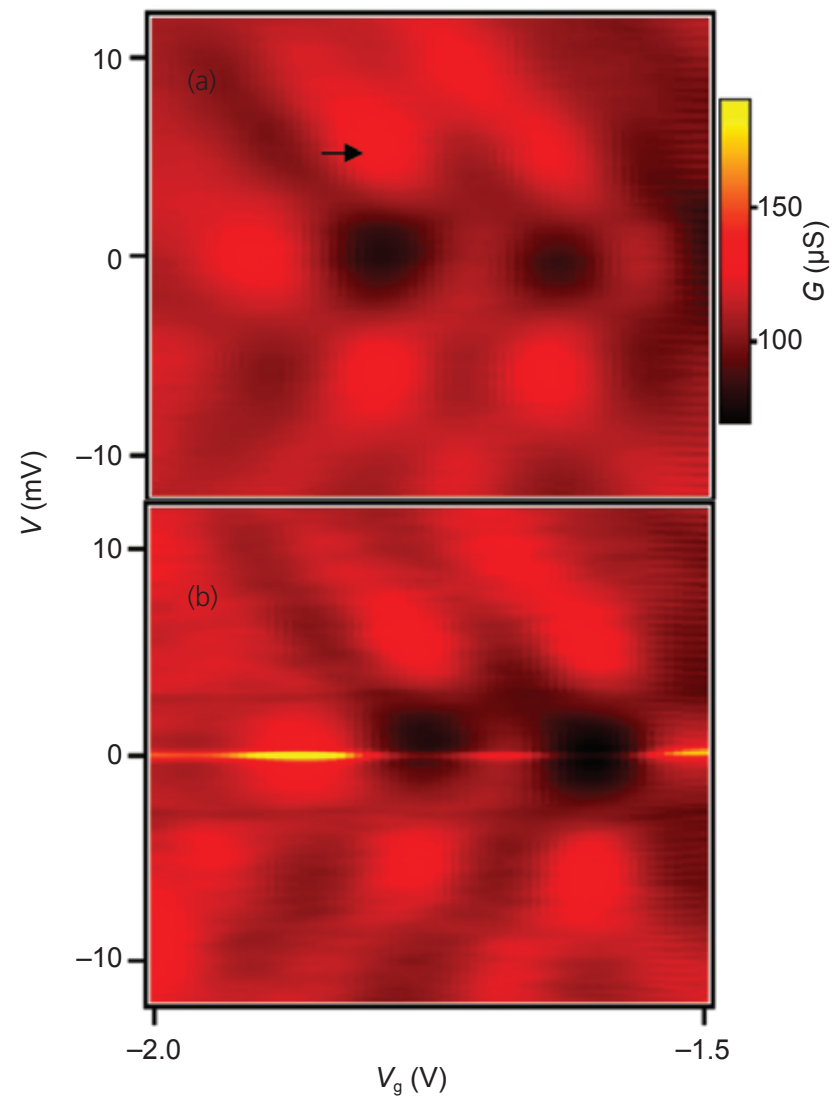

Figure 1 Device 1: differential conductance plot as a function of bias and gate voltage (a) with a magnetic field of $8 \mathrm{~T}$ and (b) without magnetic field. The arrow in (a) indicates the characteristic voltage of Fabry-Perot interference through resonant and off-resonant states (Fig. 1(b)). For Device 2 with its thinner Pd contact layer, we observed giant zero-bias conductance, which reached as high as $10 e^{2} / h$, much larger than $G_{0}$, indicative of a superconducting proximity effect (Fig. 2). At finite but small biases, several pronounced conductance peaks were observed. We identify $2 \Delta / e= \pm 0.18 \mathrm{mV}$, where the conductance peaks at $2 \Delta$ correspond to the onset of direct quasiparticle transport ( $\Delta$ is the superconducting gap of $\mathrm{Al}$ ). The peaks at $V \leqslant \pm 0.09 \mathrm{mV}$ result from MAR processes [21]. During an Andreev reflection, an incident electron at the SWNT/S interface is reflected as a hole, with the formation of a Cooper pair in a superconducting condensate. For a S/SWNT/S junction, an electron can be reflected back and forth between the electrodes several times, each time gaining energy eV, before it gathers sufficient energy to exit the SWNT as a quasiparticle into the superconductor. MAR processes give rise [22] to features in $\mathrm{d} I / \mathrm{d} V$ at voltages which are subharmonic multiples of $2 \Delta$, and contribute to the giant conductance peak at zero-bias. As shown in Fig. 2, the MAR features persist throughout the whole range of measured gate voltage, with the position of peaks fluctuating slightly with changing $V_{\mathrm{g}}$.

To investigate the possibility of a supercurrent, we

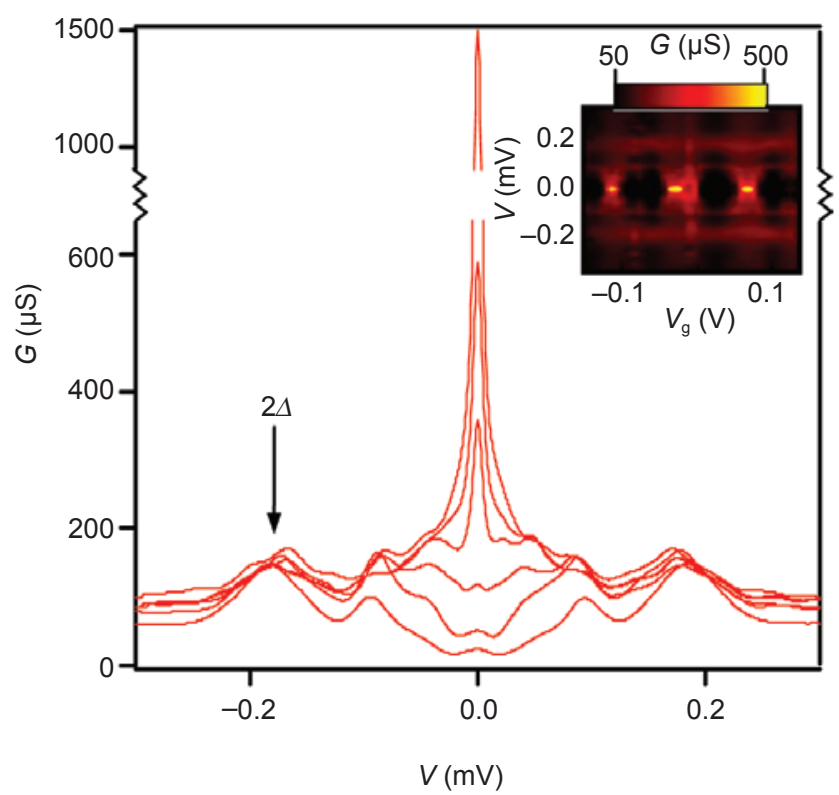

Figure 2 Plot of conductance $G_{v s}$ bias voltage $V$ for Device 2 at $V_{9}=0.024,0.044,0.002,-0.041,-0.038$, and $-0.029 \vee$ (from bottom to top at $V=0$ ). The inset shows the MAR features, plotting $G$ as a function of bias and gate voltages 
current-biased the devices and the resulting dc $V-I$ characteristics are shown in Fig. 3(a). At low current I (below the order of $\mathrm{nA}$ ), the devices remain on the supercurrent branch and display finite (and typically small) linear response resistance $R_{0}$; when the bias current exceeds a threshold, $I_{\mathrm{s}}$ the measured voltage abruptly switches to the quasiparticle branch, with a resistance that approaches $R_{\mathrm{N}}$, the normal state resistance of the junction. The $V-I$ characteristics are strongly gate-dependent: both the switching current $I_{\mathrm{s}}$ and linear response resistance $R_{0}$ can be modulated by $V_{\mathrm{g}}$ and are correlated with the normal

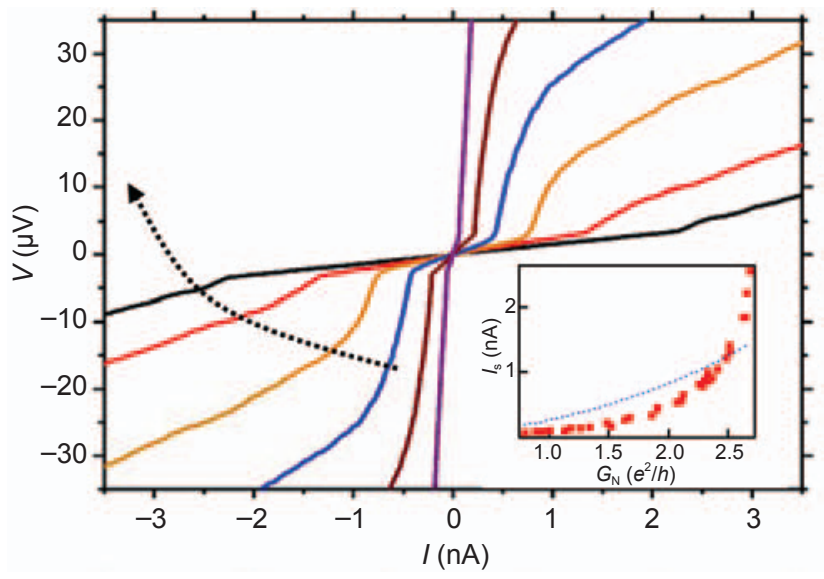

(a)

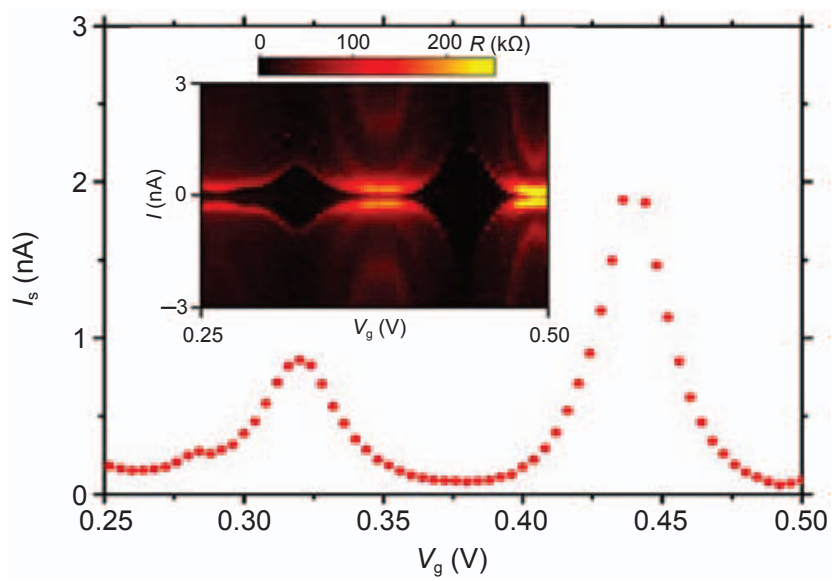

(b)

Figure 3 (a) $V-I$ characteristics showing the modulation of the switching current $I_{s}$ with values of $V_{g}$ (increasing in the direction of the arrow) of $0.485,0.479,0.473,0.467,0.445$ and $0.440 \mathrm{~V}$. The inset displays a plot of the switching current vs normal state conductance $G_{N}$. The dotted line is a best fit to Eq. (2a) with the fitting parameter $I_{c}=5.2 \mathrm{nA}$. (b) Plot of switching current vs gate voltage at two resonant states. The differential resistance plot is shown in the inset state conductance $G_{\mathrm{N}}$ (Fig. 3(a) inset, Fig. 3(b)). For instance, for the $V_{\mathrm{g}}$ range $0.44-0.536 \mathrm{~V}, G_{\mathrm{N}}$ decreases from $2.68 e^{2} / h$ to $0.8 e^{2} / h, I_{\mathrm{s}}$ decreases from about 2.5 $\mathrm{nA}$ to $65 \mathrm{pA}$, while $R_{0}$ increases from about $1.5 \mathrm{k} \Omega$ up to $44 \mathrm{k} \Omega$. Moreover, there exists a simple relationship between $I_{\mathrm{s}}$ and $R_{0}$. In a log-log plot of $I_{\mathrm{s}}$ and $R_{0}$ (Fig. 4 inset) the data points fall on a straight line, indicating a power-law dependence. The solid line is a bestfit curve to $I_{\mathrm{s}}=A / R_{0}$, with the value of coefficient $A$ being $\sim 3200 \mathrm{nA} \cdot \Omega$.

Gate-tunable $V-I$ characteristics and supercurrent have been observed [5, 9] in SWNTs, arising from resonant and off-resonant transport across quantized single particle level in a finite SWNT segment. Theoretically [23], for two superconductors symmetrically coupled via discrete energy levels in a 2-channel quantum dot, the maximum critical current in the wide resonance regime $(I \gg \Delta)$ is

$$
I_{\mathrm{c} 0} \approx \frac{2 e \Delta}{h} \tanh \left(\frac{\Delta}{2 k_{\mathrm{B}} T}\right)
$$

where $T=300 \mathrm{mK}$ is the temperature, $\Gamma \sim 1 \mathrm{meV}$ is the level broadening due to the finite lifetime of the electron $^{1}, e$ is the electron charge. For Device 2, $2 \Delta \sim 0.18$

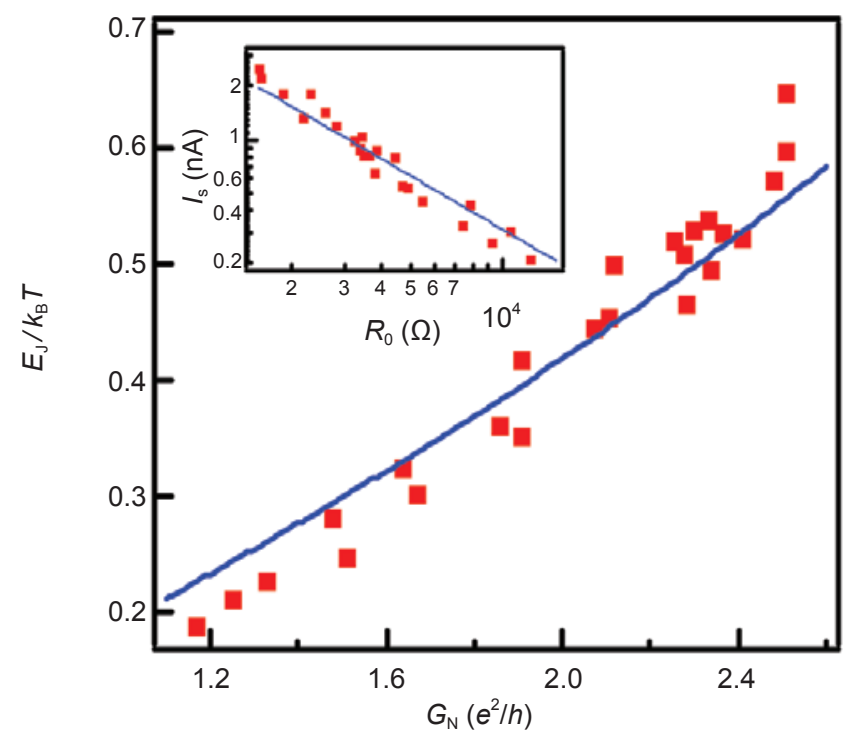

Figure 4 Main panel: a plot of $\frac{E_{J}}{k_{\mathrm{B}} T}$ (see text) vs normal state conductance. Red squares are data points and the solid line is a fit to Eq. (5). Inset: Plot of switching current vs. zero bias resistance. Red squares are data points and the solid line is fit to the data using $I_{s}=\frac{A}{R_{0}}$, where $A$ is a fitting parameter, determined to be $3200 \mathrm{nA} \cdot \Omega$

\footnotetext{
${ }^{1}$ For SWNTs in the Fabry-Perot regime, $\Gamma$ is on the order of level spacing i.e., meV. A more quantitative estimate of $\Gamma$ can be obtained by fitting the $G_{\mathrm{N}}\left(V_{\mathrm{g}}\right)$ curve to the Breit-Wigner transmission line shape (see e.g., Ref. [5] for details).
} 
meV as determined from the MAR features, yielding $I_{\mathrm{c} 0} \sim 41 \mathrm{nA}$. In actual devices, the asymmetric coupling is expected to decrease the measured normal state conductance $G_{\mathrm{N}}$, which in turn leads to a reduction in the actual critical current, given by

$$
I_{\mathrm{c}}=I_{\mathrm{c} 0}\left(1-\sqrt{1-\frac{G_{\mathrm{N}}}{4 e^{2} / h}}\right)
$$

The maximum value of $G_{\mathrm{N}}$ is $2.68 e^{2} / h$ for Device 2, and thus we expect the critical current to be as large as $\sim 17.4 \mathrm{nA}$. This value is nearly an order of magnitude larger than the observed value of $2.5 \mathrm{nA}$. This large discrepancy cannot be accounted for by the presence of disorders, since in this case $I_{\mathrm{c}}$ would be expected [24] to be lower but remain on the same order of magnitude as given by Eq. (2).

To understand the inverse relationship $I_{\mathrm{s}} \propto R_{0}^{-1}$ and the large discrepancy between theoretical and experimental values of the critical current, we focus on the dynamics of the SWNT Josephson junction within the RCSJ model. The dc $V-I$ characteristics of the devices are consistent with that of an underdamped junction [14]. In this case, small fluctuations from the thermal or electromagnetic environment leads to premature switching of the junction into the "running" or resistive state, yielding $[5,25]$

$$
I_{\mathrm{s}}=I_{\mathrm{c}}\left(1-\sqrt{1-\frac{G_{\mathrm{N}}}{4 e^{2} / h}}\right)^{3 / 2}
$$

Equation (2a) can qualitatively account for the monotonic decrease in $I_{\mathrm{s}}$ with $G_{\mathrm{N}}$. However, fitting the data $I_{\mathrm{s}}\left(G_{\mathrm{N}}\right)$ to Eq. (2a) results in poor quantitative agreement (Fig. 3(a) inset, dotted line). Thus the simple picture of premature switching in underdamped junctions does not adequately account for our data.

Here we show that the observed behavior can be quantitatively described by the classical phase diffusion model $[15,16]$. In the phase diffusion regime, the junction dynamics depends crucially on the impedance $Z$ of the biasing circuit at the characteristic plasma frequency of the JJ. Since the junction is not isolated from its electromagnetic environment, $Z$ is typically on the order of the impedance of free space ( $377 \Omega$ ) (the exact value of $Z$ depends on the detailed circuit configuration). Hence, even though the junction is underdamped at low frequencies, it is overdamped at high frequencies, which causes the "particle" (i.e., the relative phase across the junction) to diffuse (instead of running) down the tilted washboard potential. This leads to a finite voltage measured in the nominally zero resistance state, yielding [16] a zero-bias resistance

$$
R_{0}=\frac{Z}{I_{0}^{2}\left(E_{\mathrm{J}} / k_{\mathrm{B}} T\right)-1}
$$

where $E_{\mathrm{J}}=\frac{h I_{\mathrm{c}}}{2 e}$ is the Josephson coupling energy of the junction, $Z$ is the environmental impedance at ac, and $I_{0}(x)$ is the modified Bessel function. For Device 2, the upper limit of $E_{\mathrm{J}}$ is estimated to be $\sim 0.036 \mathrm{meV}$ for $I_{\mathrm{c}} \sim 18 \mathrm{nA}$, comparable to the thermal energy $k_{\mathrm{B}} T$ $\sim 0.025 \mathrm{meV}$ at $300 \mathrm{mK}$. We thus consider the weak Josephson coupling regime of $E_{\mathrm{J}}<k_{\mathrm{B}} T$, where Eq. (3) is simplified to

$$
R_{0}=2 Z\left(\frac{k_{\mathrm{B}} T}{E_{\mathrm{J}}}\right)^{2}
$$

and the switching current is given by $I_{\mathrm{s}}=\frac{e E_{\mathrm{J}}^{2}}{2 k_{\mathrm{B}} T h}$. Combining the two equations yields $I_{\mathrm{s}}=\frac{e Z k_{\mathrm{B}} T}{h} \frac{1}{R_{0}}$. Thus, $I_{\mathrm{s}}$ is proportional to $R_{0}^{-1}$, in agreement with our experimental observation (Fig. 4 inset). Substituting the fitting coefficient $A=3200 \mathrm{nA} \cdot \Omega$ into $\frac{e Z k_{\mathrm{B}} T}{h}$, gives a value of $Z \sim 485 \Omega$, which is a reasonable value. (Here five data points with $R_{0}>R_{\mathrm{N}}$ were excluded from the fitting, because the large values of $R_{0}$ indicate reduced quasiparticle conductance due to the formation of the superconducting energy gap in the density of states, where Eqs. (3) and (4) are inapplicable.)

Further insight is provided by investigating the dependence of $R_{0}$ on $G_{\mathrm{N}}$ at different gate voltages. For each $V_{\mathrm{g}}$, we first calculate the ratio $\frac{E_{\mathrm{J}}}{k_{\mathrm{B}} T}$ by solving Eq. (3) numerically using the measured values of $R_{0}$ and $Z=485 \Omega$. The calculated values of $\frac{E_{\mathrm{J}}}{k_{\mathrm{B}} T}$ are then plotted against $G_{\mathrm{N}}$ (in units of $e^{2} / h$ ) in Fig. 4. From Eq. (2), we expect

$$
\frac{E_{\mathrm{J}}}{k_{\mathrm{B}} T}=\frac{h I_{\mathrm{c} 0}}{2 e k_{\mathrm{B}} T}\left(1-\sqrt{1-\frac{G_{\mathrm{N}}}{4 e^{2} / h}}\right)
$$

We fitted Eq. (5) to the data points with $I_{\mathrm{c} 0}$ as the fitting parameter, and obtained reasonable agreement 
between experimental data (red squares) and calculation (blue line). The fitting yields $I_{\mathrm{c} 0}=21 \mathrm{nA}$, $51 \%$ of the ideal value of $\sim 41 \mathrm{nA}$. This reduction can be attributed [24] to possible defects in Device 2. Thus, our data are well described by the phase diffusion model in the weak Josephson coupling regime.

In summary, we observe proximity effectinduced superconductivity in S/SWNT/S Josephson junctions, in which the MAR processes and the supercurrent features can be tuned by the gate voltage. The finite zero bias resistance $R_{0}$ and magnitude of the switching current $I_{\mathrm{s}}$ in the $V-I$ characteristics are in good agreement with the phase diffusion model in RCSJ.

\section{References}

[1] Morpurgo, A. F.; Kong, J.; Marcus, C. M.; Dai, H. Gatecontrolled superconducting proximity effect in carbon nanotubes. Science 1999, 286, 263-265.

[2] Kasumov, A. Y.; Deblock, R.; Kociak, M.; Reulet B; Bouchiat, H.; khodos, I. I.; Gorbatov, Y. B.; Volkov, V. T.; Journet, C.; Burghard, M. Supercurrents through singlewalled carbon nanotubes. Science 1999, 284, 15081511.

[3] Buitelaar, M. R.; Nussbaumer, T.; Schonenberger, C. Quantum dot in the Kondo regime coupled to superconductors. Phys. Rev. Lett. 2002, 89, 256801.

[4] Buitelaar, M. R.; Belzig, W.; Nussbaumer, T.; Babic, B.; Bruder, C.; Schonenberger, C. Multiple Andreev reflections in a carbon nanotube quantum dot. Phys. Rev. Lett. 2003, 91, 057005.

[5] Jarillo-Herrero, P.; van Dam, J. A.; Kouwenhoven, L. P. Quantum supercurrent transistors in carbon nanotubes. Nature 2006, 439, 953-956.

[6] Jorgensen, H. I.; Grove-Rasmussen, K.; Novotny, T.; Flensberg, K.; Lindelof, P. E. Electron transport in singlewall carbon nanotube weak links in the Fabry-Perot regime. Phys. Rev. Lett. 2006, 96, 207003.

[7] Cleuziou, J. P.; Wernsdorfer, W.; Bouchiat, V.; Ondarcuhu, T.; Monthioux, M. Carbon nanotube superconducting quantum interference device. Nat. Nanotechnol. 2006, 1, 53-59.

[8] Cleuziou, J. P.; Wernsdorfer, W.; Andergassen, S.; Florens, S.; Bouchiat, V.; Ondarcuhu, T.; Monthioux, M.
Gate-tuned high frequency response of carbon nanotube Josephson junctions. Phys. Rev. Lett. 2007, 99, 117001.

[9] Tsuneta, T.; Lechner, L.; Hakonen, P. J. Gate-controlled superconductivity in a diffusive multiwalled carbon nanotube. Phys. Rev. Lett. 2007, 98, 087002.

[10] Eichler, A.; Weiss, M.; Oberholzer, S.; Schonenberger, C.; Yeyati, A. L.; Cuevas, J. C.; Martin-Rodero, A. Even-odd effect in Andreev transport through a carbon nanotube quantum dot. Phys. Rev. Lett. 2007, 99, 126602.

[11] Jorgensen, H. I.; Novotny, T.; Grove-Rasmussen, K.; Flensberg, K.; Lindelof, P. E. Critical current 0-pi transition in designed Josephson quantum dot junctions. Nano Lett. 2007, 7, 2441-2445.

[12] Sahoo, S.; Kontos, T.; Furer, J.; Hoffmann, C.; Graber, M.; Cottet, A.; Schonenberger, C. Electric field control of spin transport. Nat. Phys. 2005, 1, 99-102.

[13] Man, H. T.; Wever, I. J. W.; Morpurgo, A. F. Spindependent quantum interference in single-wall carbon nanotubes with ferromagnetic contacts. Phys. Rev. B 2006, 73, 241401.

[14] Tinkham, M. Introduction to Superconductivity. Second Edition; McGraw-Hill Book Co.: New York, 1996.

[15] Martinis, J. M.; Kautz, R. L. Classical phase diffusion in small hysteretic Josephson junctions. Phys. Rev. Lett. 1989, 63, 1507-1510.

[16] Ingold, G. L.; Grabert, H.; Eberhardt, U. Cooper-pair current through ultrasmall Josephson junctions. Phys. Rev. B 1994, 50, 395-402.

[17] Kong, J.; Soh, H. T.; Cassell, A. M.; Quate, C. F.; Dai, H. J. Synthesis of individual single-walled carbon nanotubes on patterned silicon wafers. Nature 1998, 395, 878881.

[18] Liang, W. J.; Bockrath, M.; Bozovic, D.; Hafner, J. H.; Tinkham, M.; Park, H. Fabry-Perot interference in a nanotube electron waveguide. Nature 2001, 411, 665669.

[19] Zhou, S. Y.; Gweon, G. H.; Graf, J.; Fedorov, A. V.; Spataru, C. D.; Diehl, R. D.; Kopelevich, Y.; Lee, D. H.; Louie, S. G.; Lanzara, A. First direct observation of Dirac fermions in graphite. Nat. Phys. 2006, 2, 595-599.

[20] Lemay, S. G.; Janssen, J. W.; van den Hout, M.; Mooij, M.; Bronikowski, M. J.; Willis, P. A.; Smalley, R. E.; Kouwenhoven, L. P.; Dekker, C. Two-dimensional imaging of electronic wavefunctions in carbon nanotubes. Nature 2001, 412, 617-620.

[21] Andreev, A. F. The thermal conductivity of the 
intermediate state in superconductors. Sov. Phys. JETP 1964, 19, 1228.

[22] Blonder, G. E.; Tinkham, M.; Klapwijk, T. M. Transition from metallic to tunneling regimes in superconducting micro-constrictions-excess current, charge imbalance, and super-current conversion. Phys. Rev. B 1982, 25, 451.

[23] Beenakker, C. W. J.; van Houten, H. Resonant Josephson current through a quantum dot. In Single Electron Tunneling and Mesoscopic Devices, Koch, H.; Lubbig, H.,
Eds. Springer: Berlin, 1992; pp 175-179.

[24] Beenakker, C. W. J. Three "universal" mesoscopic Josephson effects. In Transport Phenomena in Mesoscopic Systems, Fukuyama, H.; Ando, T., Eds. Springer: Berlin, 1992.

[25] Joyez, P.; Lafarge, P.; Filipe, A.; Esteve, D.; Devoret, M. H. Observation of parity-induced suppression of Josephson tunneling in the superconducting single electron transistor. Phys. Rev. Lett. 1994, 72, 2458-2461. 